\title{
The Matter and Energy Transfer Mechanism in Industrial Clusters Innovation Ecosystem
}

\author{
Xizhan YU, Chuanbo Zhang \\ Shandong University of Science and Technology, Qingdao, Shandong Province China \\ yxzsd@126.com,2320771606@qq.com
}

\begin{abstract}
With the natural ecosystems, the survival and development in industrial clusters innovation ecosystem, also need mater and energy. The major material and energy, such as knowledge, talent , capital etc., handing over with each other function, together constitute the complicated structure of the system. The center is enterprise and the supports are universities, governments and intermediary institutions, etc. The mater and energy are passed along different paths among the center, supports and the external environment. On the influence of formal and informal communication mechanisms, the process of energy transfer in the system achieve value increment of innovative knowledge, by which innovation will be constantly produced and innovation offset will also be get at the same time. Furthermore, innovation ecosystem will gain more and more energy to achieve survival and sustainable development.
\end{abstract}

Index Terms - Industrial clusters, Innovation ecosystem, Matter and energy, Transfer mechanism

\section{Introduction}

To achieve survival and sustainable development, the clusters innovation system has to build appropriate nutrition network as natural ecosystems. The process of innovation essentially is a learning process, which mainly based on knowledge flow and knowledge sharing among the biological communities of the industrial cluster innovation Ecosystem. In the process, the innovation matter and energy, such as knowledge, talent, capital etc., will be frequently propagated and recycled among the enterprises, universities, governments, intermediary institutions and external environment. As a result, the innovation knowledge will be shared and proliferated to generate knowledge spillovers. The innovation activities will be constantly produced and innovation offsets will also be get at the same time. Furthermore, innovation ecosystem will gain more and more energy to achieve survival and sustainable development.

\section{The matter and Energy of Industrial Clusters Innovation Ecosystem}

The nutrient cycle and energy flow are the basic functions of the ecosystem. In industrial cluster innovation ecosystem, not only are both the matter and energy and their rules similar to the laws of natural ecosystems, also have their own uniqueness.

As the heart role of the system, technology and innovation are often characterized by production, storage, use and consumption of knowledge and information. The process of innovation essentially is a learning process, which mainly based on knowledge flow and knowledge sharing among the populations of Ecosystem. Thus, what is transferred among the biological communities is not food, but the knowledge. In addition, as the important factors that affect regional innovation ability, the innovation investment and talents are the important matter and energy of ecosystem.

In the natural ecosystem, energy flow and nutrient cycle are carried out by the means of feeding among biotic communities. Material flow is conserved loop, but energy flow is unidirectional. Flowing in the ecosystem, the energy will be dissipated as thermal energy and constantly be wound down. But in the innovation ecosystems, the matter and energy are continually being enhanced (As LABLE1).

TABLE.1 The comparison of matter and energy

\begin{tabular}{|c|c|l|c|c|}
\hline \multirow{2}{*}{} & \multicolumn{2}{|c|}{ Natural ecosystem } & \multicolumn{2}{c|}{ Innovation ecosystem } \\
\cline { 2 - 5 } & Constituent & Characteristic & Constituent & Characteristic \\
\hline Matter & $\begin{array}{c}\text { Oxygen, carbon, } \\
\text { hydrogen, } \\
\text { nitrogen, } \\
\text { phosphorus, etc. }\end{array}$ & Conservation & $\begin{array}{c}\text { Talent, } \\
\text { capital }\end{array}$ & $\begin{array}{c}\text { Enhancing } \\
\text { continually }\end{array}$ \\
\hline Energy & Thermal energy & $\begin{array}{c}\text { Stage } \\
\text { sheddingtm }\end{array}$ & $\begin{array}{c}\text { Knowledge, } \\
\text { value }\end{array}$ & $\begin{array}{c}\text { Enhancing } \\
\text { continually }\end{array}$ \\
\hline
\end{tabular}

\section{The Structure of Matter and Energy}

In natural ecosystem, everything is interconnection and interacts with each other by way of food chain and food network. But in the industrial cluster innovation ecosystems, the structure of matter and energy is the innovation network that connects with the main communities, such as enterprises, universities, research institutions, intermediaries, government, etc. Although various ecosystem communities character and function differently in the innovation processes, various food structures are eventually formed by way of direct or indirect contacts and cooperation. The trophicre lation is the key channel to transfer knowledge, information and technology, and is the value chain to create value and make added value possible.

Among them, enterprise, university and research institution are the most important innovation bodies, the cooperation with each other and the frequency of knowledge flow will effect overall ability to innovate. In the cluster innovation ecosystem, the key to continuously improve the innovation function is whether to have some high level of universities and research institutions or to establish close contact with them. The practices of developed countries and newly industrialized economies commonly prove that 
universities and research institutions play the core leading roles in the innovation process. In the world, some of famous high-tech industrial clusters and Sci-tech Parks have been developed on the famous universities and research institutes (As TABLE 2).

TABLE.2 The comparison of knowledge source in the world famous cluster ecosystem

\begin{tabular}{|l|l|l|}
\hline $\begin{array}{l}\text { Cluster } \\
\text { innovation } \\
\text { ecosystem }\end{array}$ & Universities & $\begin{array}{l}\text { Research } \\
\text { institutions }\end{array}$ \\
\hline Silicon valley & $\begin{array}{l}\text { Stanford University, University } \\
\text { of California, etc }\end{array}$ & Bell Labs \\
\hline $\begin{array}{l}\text { Boston 128 road } \\
\text { area }\end{array}$ & $\begin{array}{l}\text { MIT, Harvard university, Yale } \\
\text { University,etc. }\end{array}$ & $\begin{array}{l}\text { Lincoln } \\
\text { experiment center }\end{array}$ \\
\hline $\begin{array}{l}\text { Singapore science } \\
\text { park }\end{array}$ & $\begin{array}{l}\text { National university of Singapore, } \\
\text { Singapore polytechnic university, } \\
\text { Nanyang Polytechnic, etc. }\end{array}$ & $\begin{array}{l}\text { Standards and } \\
\text { industry research } \\
\text { institute }\end{array}$ \\
\hline $\begin{array}{l}\text { Zhongguancun } \\
\text { Sci-tech Parks }\end{array}$ & $\begin{array}{l}\text { Tsinghua university, Peking } \\
\text { University, etc }\end{array}$ & $\begin{array}{l}\text { Chinese academy } \\
\text { of cciences }\end{array}$ \\
\hline $\begin{array}{l}\text { Taiwan hsinchu } \\
\text { industrial park }\end{array}$ & $\begin{array}{l}\text { "National Tsing Hua University", } \\
\text { "Peter Shih" }\end{array}$ & $\begin{array}{l}\text { Industrial } \\
\text { technology } \\
\text { research Institute }\end{array}$ \\
\hline
\end{tabular}

In addition, other communities, such as users, competitors, suppliers distributor, etc., also take part in the innovation processes and play their own roles (As TABLE 3).

TABLE. 3 The relative importance of different participants in the process of knowledge flow

\begin{tabular}{|l|c|c|}
\hline Participants & $\begin{array}{c}\text { knowledge } \\
\text { acquisition } \\
\text { Users }\end{array}$ & $\begin{array}{c}\text { knowledge } \\
\text { innowation }\end{array}$ \\
\hline $\begin{array}{l}\text { University and research } \\
\text { institution } \\
\text { competitor }\end{array}$ & 3.7 & 3.9 \\
\hline $\begin{array}{l}\text { supplier } \\
\text { government }\end{array}$ & 2.9 & 3.4 \\
\hline Industry association & 3.2 & 3.0 \\
\hline Foreign enterprise & 2.6 & 3.0 \\
\hline Facilitating agencies & 2.6 & 2.8 \\
\hline
\end{tabular}

Note: the 5-point scale, where 5 is the most important, and 1 is the least important.

\section{The matter and energy transfer mechanism of cluster innovation ecosystem}

In cluster innovation ecosystem, the trophic interrelations experienced with ecosystem communities are diverse, interactive, non-linear and strong coupling. There are both the formal innovative partnerships based on the rules and cooperation agreements, and the informal communication relationships based on the social network. But the formal and informal relationships are not completely contradictory. In most cases, they cooperate with each other to transfer the matter and energy, so as to carry out innovations.

In innovation ecosystem, the formal innovative partnerships taking enterprises as the core will form a dominant character path to transfer the matter and energy. The relationships based on social network will also form an implicit informal transmission path for supporting and maintaining the official network. Correspondingly, there are formal and informal mechanisms to transfer matter and energy in the industrial cluster innovation ecosystem.

\section{A. The Formal Matter and Energy Transfer Mechanism}

Under the formal mechanism, the matter and energy can be transferred through the formal innovative network contacted with the ecosystem communities. In the process of innovation, this formal network often can be represented as a tangible and objective existence form. This is a long-term stable partnership which was formed by communities actively cooperating with other actors. For differently contacting with each other, there are various innovation networks: R\&D collaboration agreements, technology swap alliances, license agreement, the direct investment driven by technical factors, joint venture enterprise, research association, subcontracting, distribution networks and joint research project funded by the government, etc.

The interactions with ecosystem communities each other are more complicated in the industrial cluster innovation ecosystem. It could bemade known that the knowledge could be transferred interactively and networked. In the real world, any live system can be represented as the network model by means of complex network theory.

\section{B. The Informal Matter and Energy Transfer Mechanism}

On the informal communication mechanism, the matter and energy transfer usually depend on social networks contacting with people who have common social and cultural background. Generally, there are two ways for knowledge Flow and dissemination. One is the public relations network in non-market trading activities, other is private networks. The most important in informal network are the potential experienced knowledge and un-encoded knowledge which could be transferred by way of direct interaction in the process of working, living and learning. It has been shown that face to face interaction is the most effective way to circulate knowledge. For one, the knowledge obtained from his colleagues and friends is more five times than that obtained form other ways.

Under this condition, the ecosystem communities can cooperate with each other without mandatory obligation. And knowledge flow is a contact transmission that shows more characteristics similar to the spread of infectious diseases. Thus, it can be learnt to explain the flow of informal knowledge transfer mechanisms by means of the spread of infectious disease.

\section{1) Basic Assumptions:}

(1) $\mathrm{N}$ is the total number of ecosystem, and it is large enough.

At $\mathrm{T}$ time, the staff of system can be divided into three categories: the $\mathrm{H}$ class represents the owner of tacit knowledge, the $\mathrm{P}$ class represents the infected of tacit knowledge, the Q class represents the dropout. 
And the state of system can be divided into 3 types: none infected directly quits, parts of the infected of tacit knowledge quits, parts of the infected of tacit knowledge quit, parts of the owner of tacit knowledge quit.

(2) At $\mathrm{T}$ time, the ratio of three categories can be expressed as follows: $\mathrm{p}(\mathrm{t}), \mathrm{h}(\mathrm{t})$ and $\mathrm{q}(\mathrm{t})$.

(3) Forgetting factor is not be considered in the model. Because once infected people become the winner of the tacit knowledge, it is impossible to completely forgotten or disappear.

(4) When tacit knowledge holders annually effectively contacted with the infected annually, they may become the new owners of tacit knowledge. Assuming that $\lambda$ is the rate of annual effective contacting, and $1-\lambda$ is the rate of the tacit knowledge infected exiting.

(5) Assuming that $\mu$ is the rate of the owner of tacit knowledge exiting, and $\alpha$ is the rate of new entrants. To maintain the stability of system, assuming that:

$$
\alpha \mathrm{N}=(1-\lambda) \mathrm{Np}(\mathrm{t})+\mu \mathrm{Nh}(\mathrm{t})
$$

(6) Assuming that none infected person directly quit in the model.

2) Modeling Based on Assumptions:

$$
\left\{\begin{array}{l}
\frac{d p}{d t}=-\lambda p(t) h(t)+\alpha-(1-\lambda) p(t) \\
\frac{d h}{d t}=\lambda p(t) h(t)-\mu h(t) \\
\alpha=(1-\lambda) p(t)+\mu h(t) \\
H_{0}=N h\left(t_{0}\right), P_{0}=N p\left(t_{0}\right)
\end{array}\right.
$$

Then its analytical solution is as the follows:

$$
\begin{gathered}
h(t)=\frac{e^{\left(\frac{\alpha \lambda}{1-\lambda}-\mu\right) t}}{\left[\frac{1}{H}+\frac{\lambda \mu}{(1-\lambda) \mu-\alpha \lambda}\right]-\frac{\lambda \mu}{(1-\lambda) \mu-\alpha \lambda} e^{\left(\frac{\alpha \lambda}{1-\lambda}-\mu\right) t}} \\
\text { If } t \rightarrow \infty \text {,assuming that } \sigma=\left(\frac{1}{\lambda}-\frac{\alpha}{\mu}\right), \text { then } \\
\lim _{t \rightarrow \infty} h(t)=\frac{\alpha \lambda-(1-\lambda) \mu}{\lambda \mu}=1-\left(\frac{1}{\lambda}-\frac{\alpha}{\mu}\right)=1-\sigma
\end{gathered}
$$

If $\sigma>1, \quad \lambda>\frac{\mu}{\mu+\alpha}$, the infected of the tacit knowledge could constantly be turned into the owners, and the number will continue to inch up $\mathrm{N}(1-\sigma)$.

If $\sigma \leq 1, \quad \lambda \leq \frac{\mu}{\mu+\alpha}$, the number of tacit knowledge owners will be less and less, eventually close to zero.

Thus, the contact rate, $\lambda=\frac{\mu}{\mu+\alpha}$, is the threshold value to judge if the infected of tacit knowledge could be transformed into the owners.

If $\alpha$ and $\mu$ are fixed value and there is $\frac{\mu}{\mu+\alpha}=c$ (c is constant), then a conclusion can be drawn:

$$
\begin{aligned}
& \text { if } \lambda>\frac{\mu}{\mu+\alpha}=c \quad, \quad \lim _{t \rightarrow \infty} h(t)=1-\sigma \\
& \text { if } \lambda \leq \frac{\mu}{\mu+\alpha}=\mathrm{c}, \quad \lim _{t \rightarrow \infty} h(t)=0
\end{aligned}
$$

Furthermore, if the contact rate $\lambda$ was lager than $\mathrm{c}$, the owners of tacit knowledge would die out. And the larger the contact rate $\lambda$ is, the more the owners of tacit knowledge are, eventually close to $N(1-\sigma)$. On the contrary, if $\lambda$ was smaller than $\mathrm{c}$, the infected of tacit knowledge could turn into the owners because the tacit knowledge can not be transferred from the owners to the infected.

Therefore, to effectively promote the generation and flow of knowledge and to accelerate innovation frequency, it is necessary for the ecosystem to create a more relaxed, free and positive social \& culture environment, so that the people in the system can be widely contacted with each other and strengthen communication.

If $\alpha$ and $\lambda$ were constant, the conclusion could be drawn according to $\lambda=\frac{\mu}{\mu+\alpha}$, that: if $\mu<\frac{\alpha \lambda}{1-\lambda}$, then $\lim _{t \rightarrow \infty} h(t)=1-\sigma$; if $\mu \geq \frac{\alpha \lambda}{1-\lambda}$, then $\lim _{t \rightarrow \infty} h(t)=0$. It implies that the tacit knowledge disseminators are in an unstable state. Within the communication organization, if the rate of new entrants and the contact rate were constant, whether the infected could be converted into the owner of tacit knowledge was related to the owners dropout rates.

If $\mu<\frac{\alpha \lambda}{1-\lambda}$, the infected could be converted into the owners of tacit knowledge.

If $^{\mu \geq \frac{\alpha \lambda}{1-\lambda}}$, the number to owners of tacit knowledge was close to zero and it was less possible that the infected could be turned into the owners.

It is thus clear that $\mu=\frac{\alpha \lambda}{1-\lambda}$ is the boundary condition to judge whether the knowledge communication organizations are relatively stable.

\section{The Material and Energy Transfer Path}

The industrial cluster innovation ecosystem is comprised of two layers and four communities: industrial innovation community is in the core layer, the support layers are knowledge innovation community, system and mechanism innovation community and the innovation service community. By way of formal and informal communication mechanism, the communities, such as enterprise, university, research institution, government and facilitating agency, etc., interact with each other to make the innovation knowledge flow smoothly among the core layer, support layers and external environment.

\section{A. Material and Energy Transfer Within the Core}

The process of transferring innovation knowledge within the core layer in fact is the communication among interrelated enterprises in the industrial innovation community. It could be 
carried out by two ways: one is radiation, by which the innovation enterprise transfer the knowledge to other related enterprise. The other is secondary innovation. After the transferred enterprises master the innovative knowledge, they can apply to all aspects of the enterprise include of design, production and sales. Further, they may improve and enhance the innovative technology on the basis of the products and market information. Therefore, the secondary innovation has been achieved. At the same time, these enterprises also radiate the new innovative knowledge to other enterprises and feed back the information to the pioneers. Thus, the innovation performance has been further improved by this two-way flow process of innovative knowledge. The process of knowledge flow is not linear one-way, but circulation. Innovative knowledge in this cycle is not simple superposition, but has risen in a spiral in the form of multiplier effect.

\section{B. Material and Energy Transfer between the Core and the Supports}

Within the core layer, the innovative knowledge is only transferred among the community enterprises. But as enterprises interact with the subject of auxiliary layers - universities, research institutes, intermediary service organizations and governments, the knowledge will circulate from the support layers to the core.

1) Transmitted among Firms, Universities and Research Institutes. As limited innovation capacity, most enterprises, especially small and medium-sized enterprises, are often unable to independently achieve knowledge innovation. However, in order to meet with market competition and technological innovation, enterprises must seek universities and research institutions to carry out collaborative innovation. Universities and research institutions can not only accomplish technological innovation with the companies, but also accelerate innovation knowledge flows through education, training, as well as achievements transformation modes.

2) Transmitted between Firms and Intermediary Service Organizations. With Professional method to gather information and Information maintenance technology, intermediary service institutions provide innovative knowledge faster and more accurate. In order to accelerate innovation knowledge flow with the shortest path, intermediaries sometimes are licensed by suppliers to directly and accurately selecting the consumers of innovative technology. Furthermore, by providing necessary funding for innovation source enterprises, financial institutions can ensure that innovation activities would not be delayed due to not abundant capital.

3) Transmitted between Firms and Intermediary service Organizations. With advanced communication facilities and convenient traffic conditions, the governments in the ecosystem can provide the necessary infrastructure for innovative knowledge flows. In addition, with policy system including supporting industrial policy, tax policy, fiscal policy and other policies, governments can promote to absorb and utilize innovative knowledge, so as to accelerate the flow of knowledge. Good relationship between business and Government to improve government efficiency, shorten the new knowledge flows. Besides that, good relationships between business and government can also improve government efficiency and shorten the innovative knowledge flows.

\section{Interaction with Ecosystem and External Environment}

The innovative knowledge can not only circulate among the various layers within the ecosystem, but also interact with the system and external environment, so that the innovation knowledge can be transmitted more widely. One way is exchange and cooperation. As the cluster innovation ecosystem communities achieved energy exchanges within ecosystem, the knowledge would become a mature technology after integration and precipitation. This mature technology can further be copied and transmitted outside the system. In addition to the formal and informal exchanges within the system, there are other forms of economic exchange and cooperation within and outside the cluster, such as forms of more important inter-regional cooperation, technical seminars, commodity fair, etc. The other is social culture. When the cluster communicate with external regions, the knowledge is often characterized by social culture.

Not unrelated to the three energy transfer paths, but they interact with each other and influence mutually, so that they can make a joint effect on transferring performance.

\section{Conclusion}

In short, to achieve survival and sustainable development, the clusters innovation system must build appropriate nutrition network as natural ecosystems. Innovative capitals and talents are important material basis of ecosystems. Otherwise, knowledge and value compose of creative energy. Only in accordance with the formal and informal energy exchange mechanisms, could these substances be transferred with communities and the external smoothly and fluently. As a result, the innovation knowledge will be shared and proliferated to generate knowledge spillovers. The innovation activities will be constantly produced and innovation offsets will also be get at the same time. Furthermore, innovation ecosystem will gain more and more energy to achieve survival and sustainable development.

\section{References}

[1] A.E. Douglas. Symbiotic Interactions. Oxford: Oxford University Press, 1994:3

[2] Yu Xizhan, Sui Yinghui. The Industrial Clusters Ecosystem Based on City Innovation. Science \& Technology Progress and Policy, 2010(21): 56-60

[3] Yu Xizhan. Industrial Clusters Ecosystem and its Applied Research in Resource City Innovation. Shandong University of Science and Technology Doctoral Dissertation, 2011(12):45-56

[4] Li Jianjun. Silicon Valley Model and Its University-industry Innovation System. Renmin University of China Doctoral Dissertation, 2000(6): 50

[5] Si Shangqi, Cao Zhenquan, Feng Feng. Research on the Cooperation Mechanism of Institutions and Enterprises_- A Symbiotic Theory and Analytical Framework. Science of Science and Management of S \& T, 2006(06):15-19

[6] Yu Xizhan, Sui Yinghui. Study on Symbiotic Mechanism of the Industrial Clusters Ecosystem Based on City Innovation, ICEMSS-13: 2013(08) 